\title{
OPEN Plasma neurofilament light chain is associated with cognitive decline in non-dementia older adults
}

\author{
Lingxiao He ${ }^{1 \bowtie}$, John E. Morley ${ }^{2}$, Geetika Aggarwal2, ${ }^{2,3}$, Andrew D. Nguyen ${ }^{2,3}$, Bruno Vellas $^{1,4}$, \\ Philipe de Souto Barreto ${ }^{1,4} \&$ the MAPT/DSA Group*
}

Neurofilament light chain (NfL) has been associated with cognitive status in multiple neurodegenerative conditions. Studies about plasma NfL and cognitive decline in older adults are still limited. 504 older adults (median age 75 years) who expressed memory complaints were selected from the Multidomain Alzheimer's Preventive Trial (MAPT) and were classified as normal cognition (NC) or mild cognitive impairment $(\mathrm{MCl})$. Cognitive functions were measured as mini mental state examination (MMSE) and composite cognitive score (CCS) over a 4-year period. Plasma NfL was measured at the first or the second year of the MAPT. Mixed-effects linear models were performed to evaluate cross-sectional and longitudinal associations. In the whole population, higher plasma $\mathrm{NfL}$ was cross-sectionally associated with lower cognitive functions (MMSE: $\beta=-0.007,95 \%$ $\mathrm{Cl}[-0.013,-0.001]$; CCS: $\beta=-0.003,95 \% \mathrm{Cl}[-0.006,-0.001])$. In adults with $\mathrm{MCl}$, but not $\mathrm{NC}$, higher plasma NfL was associated with lower CCS at the cross-sectional level $(\beta=-0.003,95 \% \mathrm{Cl}$ $[-0.005,-0.0002])$. The upper quartile NfL group further demonstrated more over time decline in CCS $(\beta=-0.07,95 \% \mathrm{Cl}[-0.12,-0.01])$ under the $\mathrm{MCl}$ status. Plasma $\mathrm{NfL}$ can be a promising biomarker of progressive cognition decline in older adults with $\mathrm{MCl}$.

Neurofilament is a structural protein that determines axonal caliber and conduction velocity in neurons ${ }^{1}$. As one of the three neurofilament components, neurofilament light chain (NfL) has been suggested as a biomarker of axonal damage ${ }^{2}$. High cerebrospinal fluid (CSF) NfL levels have been found in patients with multiple neurodegenerative conditions such as Parkinson's disease ${ }^{3}$, Alzheimer's disease (AD) ${ }^{4}$ and frontotemporal dementia ${ }^{5}$. As an inexpensive and less invasive parameter, plasma NfL is supposed to be a substitute of CSF NfL in the evaluation of neural degeneration due to their close correlations ${ }^{6}$. Elevated plasma and CSF NfL levels are found in multiple neural degenerative disorders such as mild cognitive impairment $(\mathrm{MCI})^{7}, \mathrm{AD}^{8,9}$ and amyotrophic lateral sclerosis ${ }^{10}$. Plasma NfL also demonstrated similar effect sizes as CSF NfL in the association with over time cognitive declines among older adults (with a median age of 76) ${ }^{7}$. Cross-sectional studies have shown that older adults with MCI had higher plasma NfL than those with normal cognition (NC), and higher NfL was associated with lower cognitive functions ${ }^{8,11}$. At a longitudinal level, greater baseline plasma NfL was associated with poorer cognitive functions over time $\mathrm{e}^{7,8}$.

Despite these findings, cross-sectional and longitudinal studies of plasma NfL and cognitive functions in nondementia older adults are still limited, and with relatively small sample sizes. Contradictory results about the significance of plasma NfL difference between the NC and MCI groups were also reported in various studies ${ }^{7,8,11,12}$. Moreover, although plasma NfL has been associated with both neuroimaging measures (e.g., hippocampal volume and cortical thickness) and cognitive functions ${ }^{7}$, it is not known if such NfL-cognition association is mediated by the brain structures.

Therefore, the aim of this study is to explore both cross-sectional and longitudinal associations between plasma NfL levels and cognition in non-dementia community-dwelling older adults with a large sample size. Further exploratory analysis was performed to explore whether the associations between plasma NfL and cognitive functions were mediated by brain imaging neurodegeneration markers (e.g., white matter hyperintensities, hippocampal volume).

\footnotetext{
${ }^{1}$ Gérontopôle de Toulouse, Institut du Vieillissement, Centre Hospitalo-Universitaire de Toulouse, 37 Allées Jules Guesdes, 31000 Toulouse, France. 'Division of Geriatric Medicine, Saint Louis University School of Medicine, St. Louis, MO, USA. ${ }^{3}$ Henry and Amelia Nasrallah Center for Neuroscience, Saint Louis University, St. Louis, MO, USA. ${ }^{4}$ CERPOP, Inserm 1295, Université de Toulouse, UPS, 31000 Toulouse, France. *A list of authors and their affiliations appears at the end of the paper. ${ }^{凶}$ email: lingxiao.he@hotmail.com
} 


\begin{tabular}{|c|c|c|c|c|c|c|}
\hline & \multicolumn{2}{|c|}{ Whole population } & \multicolumn{2}{|l|}{ NC group } & \multicolumn{2}{|l|}{ MCI group } \\
\hline & Sample size & \begin{tabular}{|l|} 
(\%) or \\
Mean (SD) or \\
Median [P25, P75]
\end{tabular} & Sample size & \begin{tabular}{|l|}
$\mathrm{N}(\%)$ or \\
Mean (SD) or \\
Median [P25, P75]
\end{tabular} & Sample size & $\begin{array}{l}\mathrm{N}(\%) \text { or } \\
\text { Mean (SD) or } \\
\text { Median [P25, P75] }\end{array}$ \\
\hline Female & 504 & $303(60 \%)$ & 223 & $145(65 \%)$ & 281 & $158(56 \%)^{*}$ \\
\hline \multicolumn{7}{|l|}{ MAPT groups } \\
\hline $\begin{array}{l}\text { Multidomain train- } \\
\text { ing+omega-3 sup- } \\
\text { plementation }\end{array}$ & 504 & $129(26 \%)$ & 223 & $49(22 \%)$ & 281 & $80(28 \%)$ \\
\hline $\begin{array}{l}\text { Omega-3 supplemen- } \\
\text { tation }\end{array}$ & 504 & $118(23 \%)$ & 223 & $53(24 \%)$ & 281 & $65(23 \%)$ \\
\hline Multidomain training & 504 & $124(25 \%)$ & 223 & $55(25 \%)$ & 281 & $69(25 \%)$ \\
\hline Placebo & 504 & $133(26 \%)$ & 223 & $66(30 \%)$ & 281 & $67(24 \%)$ \\
\hline $\begin{array}{l}\text { Participants had NfL } \\
\text { tested from first year } \\
\text { blood samples }\end{array}$ & 504 & $465(92 \%)$ & 223 & $213(96 \%)$ & 281 & $252(90 \%)$ \\
\hline Age & 504 & $75.0[72.0,79.0]$ & 223 & $74.0[71.0,78.0]$ & 281 & $76.0[72.0,79.0]^{*}$ \\
\hline Initial BMI & 501 & $26.0[23.7,28.7]$ & 220 & $26.1[24.0,29.2]$ & 281 & $25.9[23.4,28.1]$ \\
\hline $\begin{array}{l}\text { Initial plasma NfL } \\
(\mathrm{pg} / \mathrm{ml})\end{array}$ & 504 & $72.9[56.9,91.8]$ & 223 & $71.8[56.7,89.7]$ & 281 & $72.9[56.9,93.9]$ \\
\hline Initial MMSE & 502 & $28.0[27.0,79.0]$ & 223 & $29.0[28.0,30.0]$ & 279 & $28.0[26.0,29.0]^{* *}$ \\
\hline $\begin{array}{l}\text { Initial MMSE orienta- } \\
\text { tion }\end{array}$ & 502 & $10.0[10.0,10.0]$ & 223 & $10.0[10.0,10.0]$ & 279 & $10.0[9.0,10.0]^{* *}$ \\
\hline Initial FCSRT & 501 & $76.0[70.0,81.0]$ & 223 & $79.0[74.0,83.0]$ & 278 & $73.0[66.0,79.0]^{* *}$ \\
\hline Initial DSST-WAISR & 499 & $37.5(10.1)$ & 223 & $39.6(10.2)$ & 276 & $36.0(13.5)^{* *}$ \\
\hline Initial category naming & 501 & $25.5(7.7)$ & 223 & $27.2(7.1)$ & 278 & $24.0(10.0)^{* *}$ \\
\hline Initial CCS & 499 & $0.17[-0.27,0.55]$ & 223 & $0.37[0.01,0.70]$ & 276 & $-0.04[-0.61,0.48]^{* *}$ \\
\hline
\end{tabular}

Table 1. Descriptive data. BMI body mass index, CCS composite cognitive score, FCSRT free and cued selective reminding test, $N C$ normal cognition $(\mathrm{CDR}=0), M C I$ mild cognitive impairment $(\mathrm{CDR}=0.5), M M S E$ mini-mental state examination, NfL neurofilament light chain, DSST-WAISR Digit Symbol Substitution Test score from the Wechsler Adult Intelligence Scale-Revised. ${ }^{*} p<0.05,{ }^{\star *} p<0.01$ compared with the NC group.

\section{Results}

Descriptive data of the participants are presented in Table 1. Among the 504 participants (median age 75 years) included in this study, $60 \%$ were female and the median plasma NfL level was $72.9 \mathrm{pg} / \mathrm{ml}$ (with an interquartile range [IQR] of 56.9 to $91.8 \mathrm{pg} / \mathrm{ml}$ ). The MCI group included 281 participants and the NC group included 223 participants. The MCI group showed slightly higher plasma NfL levels than the NC group, but the difference was not significant. The MCI group were older than the NC group and demonstrated lower cognitive scores (Table 1). In the MCI group, the median value of initial mini mental state examination (MMSE) was 28 (IQR: $[26,29])$ and the median value of initial composite cognitive score (CCS) was -0.04 (IQR: $[-0.61,0.48]$ ). In the NC group, the median value of initial MMSE was 29 (IQR: $[28,30]$ ) and the median value of initial CCS was 0.37 (IQR: $[0.01,0.70])$.

At the cross-sectional level, plasma NfL demonstrated negative association with MMSE $(\beta=-0.007,95 \%$ CI $[-0.013,-0.001]$, Fig. 1a), digit symbol substitution test of the Wechsler adult intelligence scale-revised (DSSTWAISR) $(\beta=-0.04,95 \%$ CI $[-0.07,-0.01]$, Fig. $1 b)$ and CCS $(\beta=-0.003,95 \%$ CI $[-0.006,-0.001]$, Fig. 1c) in the whole population (Table 2), but no association was found in the NC group (Table 2). In the MCI group, higher plasma NfL levels were associated with lower DSST-WAISR $(\beta=-0.04,95 \%$ CI [ $-0.07,-0.01]$, Fig. 1d) and CCS scores $(\beta=-0.003,95 \%$ CI $[-0.005,-0.0002]$, Fig. 1e) (Table 2). No longitudinal associations were found between plasma NfL and cognitive functions in either cognitive groups.

Analysis using stratified NfL failed to find any cross-sectional or longitudinal association between NfL and cognitive functions in the NC group (Table 3). In MCI, the NfL+ subgroup showed lower DSST-WAISR $(\beta=-3.62,95 \%$ CI $[-6.1,-1.14]$, Fig. 1f $)$ and CCS scores $(\beta=-0.21,95 \%$ CI $[-0.42,-0.01]$, Fig. $1 \mathrm{~g})$ at the cross-sectional level, and greater over time decline in the CCS score than the NfL- subgroup (Table 3, Fig. 1h).

Additionally, after adjusting for the APOE genotype, only marginal significance was found at the cross-sectional level between NfL and CCS (in the whole population and the NC group) while in the MCI group, significant cross-sectional association was found between NfL and DSST-WAISR $(\beta=-0.04,95 \%$ CI $[-0.07,-0.01])$. No significant longitudinal NfL association with cognitive functions were found in models with the APOE genotype (Supplementary File Table 3 and 4). We further replicated the analyses only on the control group and similar results were found in the whole control group $(\beta=-0.07,95 \% \mathrm{CI}[-0.13,-0.01])$ and the MCI control group $(\beta=-0.08,95 \%$ CI $[-0.14,-0.02])$ that higher plasma NfL levels were cross-sectionally associated with lower DSST-WAISR scores (Supplementary File Table 5 and 6).

Exploratory mediation analysis $(n=176)$ was further performed using the structural equation modelling (SEM) method (Supplementary File Table 7 and 8) and demonstrated significant direct effects of plasma NfL on 
a)

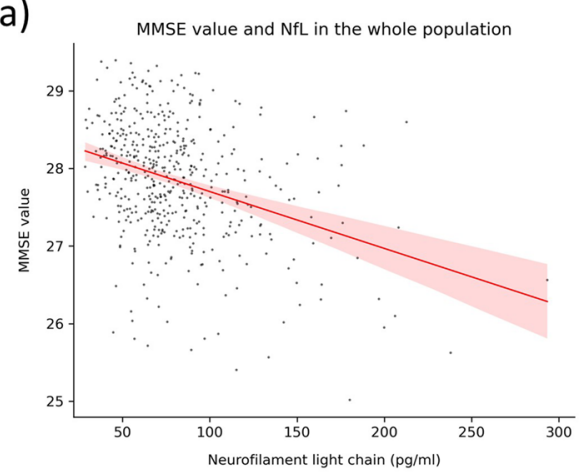

c)

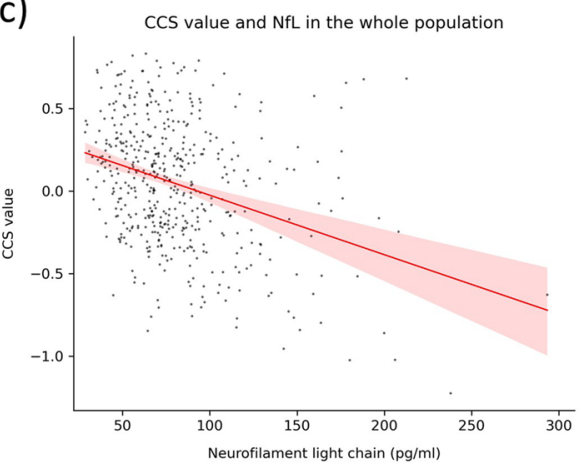

e)

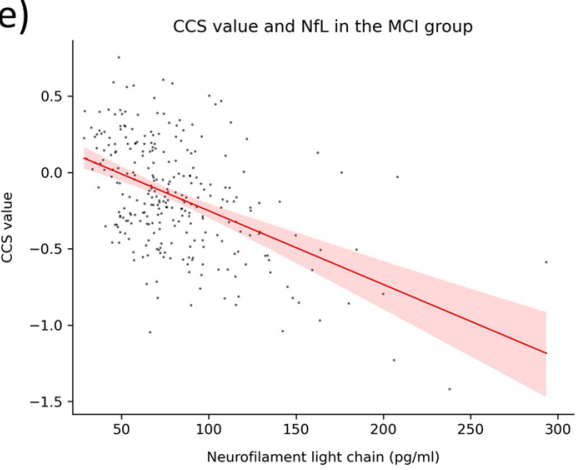

g)

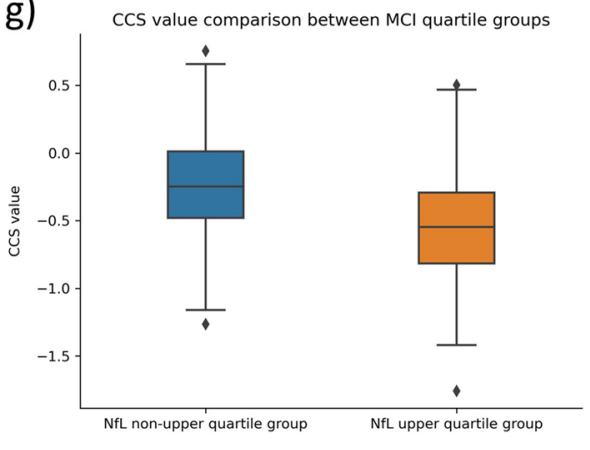

b)

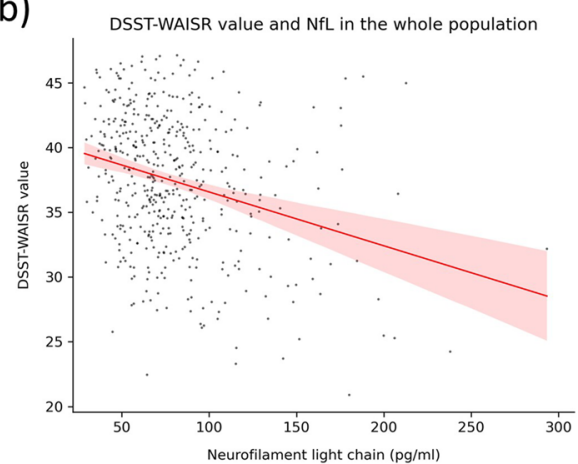

d)

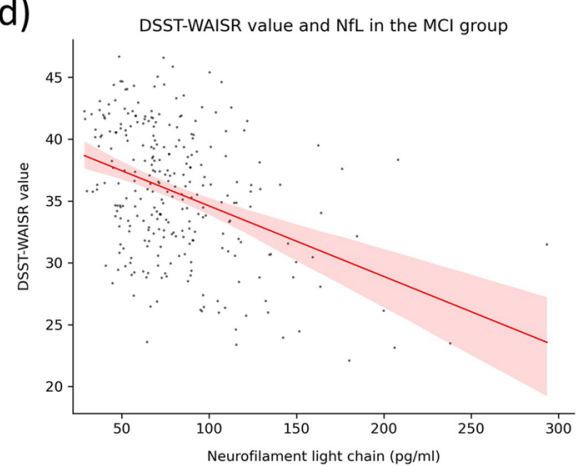

f)

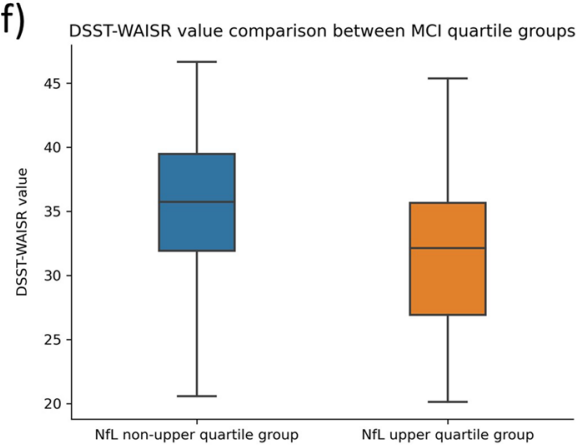

h)

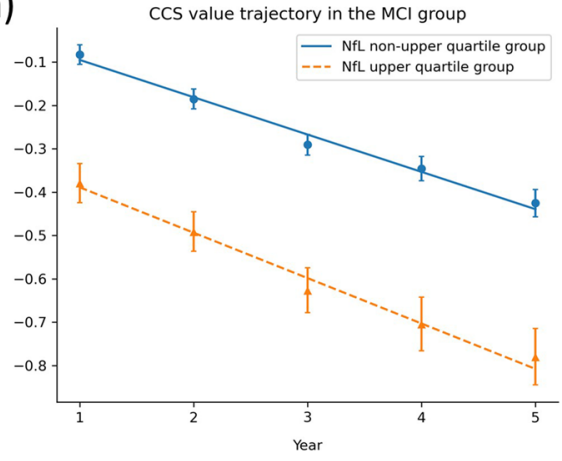

Figure 1. Significant findings in mixed-effects models. (a) Correlation between MMSE and NfL in the whole population at the initial level. (b) Correlation between DSST-WAISR and NfL in the whole population at the initial level. (c) Correlation between CCS and NfL in the whole population at the initial level. (d) Correlation between DSST-WAISR and NfL in the MCI group at the initial level. (e) Correlation between CCS and NfL in the MCI group at the initial level. (f) DSST-WAISR comparison between quartile groups in the MCI group at the initial level. (g) CCS comparison between quartile groups in the MCI group at the initial level. (h) CCS trajectory in the MCI group over a 4-year period (standard errors are used as error bars). 


\begin{tabular}{|c|c|c|c|c|c|c|c|c|c|c|}
\hline & \multirow[b]{2}{*}{ Sample size } & \multicolumn{3}{|l|}{ Initial NfL } & \multicolumn{3}{|l|}{ Time } & \multicolumn{3}{|c|}{ Initial $\mathrm{NfL} \times$ time } \\
\hline & & Coefficient & $p$ & 95\% CI & Coefficient & $p$ & 95\% CI & Coefficient & $p$ & 95\% CI \\
\hline \multicolumn{11}{|l|}{ Whole population } \\
\hline MMSE & 496 & -0.007 & 0.02 & $(-0.013,-0.001)$ & -0.129 & 0.06 & $(-0.260,0.003)$ & 0.0005 & 0.54 & \begin{tabular}{|l}
$(-0.0011$, \\
$0.0021)$
\end{tabular} \\
\hline MMSE orientation & 496 & -0.002 & 0.06 & $(-0.004,0.0001)$ & -0.019 & 0.42 & $(-0.067,0.028)$ & -0.0001 & 0.75 & $\begin{array}{l}(-0.0007 \\
0.0005)\end{array}$ \\
\hline FCSRT & 496 & -0.03 & 0.11 & $(-0.07,0.01)$ & -0.12 & $<0.001$ & $(-1.84,-0.56)$ & -0.0001 & 0.98 & $(-0.008,0.008)$ \\
\hline DSST-WAISR & 496 & -0.04 & 0.01 & $(-0.07,-0.01)$ & -0.67 & 0.01 & $(-1.14,-0.19)$ & -0.001 & 0.78 & $(-0.006,0.005)$ \\
\hline Category naming & 496 & -0.02 & 0.20 & $(-0.04,0.01)$ & -0.48 & 0.04 & $(-0.93,-0.02)$ & -0.002 & 0.46 & $(-0.007,0.003)$ \\
\hline CCS & 496 & -0.003 & 0.006 & $(-0.006,-0.001)$ & -0.07 & 0.001 & $(-0.11,-0.03)$ & -0.0003 & 0.33 & $\begin{array}{l}(-0.0008 \\
0.0003)\end{array}$ \\
\hline \multicolumn{11}{|l|}{ NC group } \\
\hline MMSE & 219 & -0.006 & 0.08 & $(-0.013,0.001)$ & -0.215 & 0.02 & $(-0.389,-0.042)$ & 0.0015 & 0.13 & \begin{tabular}{|l|}
$(-0.0005$ \\
$0.0036)$
\end{tabular} \\
\hline MMSE orientation & 219 & -0.001 & 0.29 & $(-0.003,0.001)$ & -0.04 & 0.13 & $(-0.09,0.01)$ & 0.0002 & 0.43 & $\begin{array}{l}(-0.0004 \\
0.0008)\end{array}$ \\
\hline FCSRT & 219 & -0.01 & 0.58 & $(-0.04,0.02)$ & -0.80 & 0.10 & $(-1.75,0.14)$ & -0.002 & 0.78 & $(-0.013,0.009)$ \\
\hline DSST-WAISR & 219 & -0.002 & 0.94 & $(-0.045,0.042)$ & -0.76 & 0.04 & $(-1.49,-0.03)$ & 0.001 & 0.86 & $(-0.008,0.009)$ \\
\hline Category naming & 219 & 0.01 & 0.53 & $(-0.02,0.04)$ & -0.55 & 0.12 & $(-1.24,0.14)$ & 0.0002 & 0.96 & $(-0.008,0.008)$ \\
\hline CCS & 219 & -0.0003 & 0.80 & $(-0.003,0.002)$ & -0.067 & 0.03 & $(-0.126,-0.007)$ & -0.0001 & 0.73 & $\begin{array}{l}(-0.0008 \\
0.0006)\end{array}$ \\
\hline \multicolumn{11}{|l|}{ MCI group } \\
\hline MMSE & 277 & -0.005 & 0.16 & $(-0.012,0.002)$ & -0.04 & 0.69 & $(-0.24,0.16)$ & -0.001 & 0.55 & $(-0.003,0.002)$ \\
\hline MMSE orientation & 277 & -0.001 & 0.43 & $(-0.004,0.002)$ & -0.003 & 0.93 & $(-0.082,0.075)$ & -0.0004 & 0.43 & $(-0.001,0.001)$ \\
\hline FCSRT & 277 & -0.02 & 0.29 & $(-0.07,0.02)$ & -1.51 & 0.001 & $(-2.42,-0.60)$ & 0.0004 & 0.94 & $(-0.011,0.011)$ \\
\hline DSST-WAISR & 277 & -0.04 & 0.007 & $(-0.07,-0.01)$ & -0.63 & 0.048 & $(-1.25,-0.01)$ & -0.002 & 0.60 & $(-0.010,0.006)$ \\
\hline Category naming & 277 & -0.01 & 0.44 & $(-0.04,0.02)$ & -0.32 & 0.29 & $(-0.92,0.28)$ & -0.005 & 0.18 & $(-0.012,0.002)$ \\
\hline CCS & 277 & -0.003 & 0.04 & $(-0.005,-0.0002)$ & -0.07 & 0.02 & $(-0.14,-0.01)$ & -0.0004 & 0.25 & \begin{tabular}{|l|}
$(-0.001$ \\
$0.0003)$
\end{tabular} \\
\hline
\end{tabular}

Table 2. Mixed-effects linear analysis of plasma NfL with cognitive functions. FCSRT Free and cued selective reminding test, DSST-WAISR Digit Symbol Substitution Test score from the Wechsler Adult Intelligence Scale-Revised.

DSST-WAISR and CCS in the whole population $(\mathrm{n}=176)$, and on DSST-WAISR in the MCI population $(\mathrm{n}=85)$, while no indirect effects (mediated by white matter condition and cognition-related brain structures) were found.

\section{Discussion}

The present study examined cross-sectional and longitudinal associations between plasma NfL and cognitive functions in non-dementia community-dwelling older adults (aged 70 years or above). Among adults without cognitive impairment (i.e., the NC group), plasma NfL was not associated with cognitive functions. In the MCI population, higher plasma NfL was associated with lower global cognitive scores (i.e., CCS) and executive function (i.e., DSST-WAISR) at a cross-sectional level. Moreover, MCI adults with upper quartile NfL levels demonstrated greater over time declines in the CCS score. Exploratory analysis using SEM failed to find any mediation effect of brain structures. Together, our results support the idea of using plasma NfL as a marker of predicting cognitive decline in MCI individuals ${ }^{13}$.

Based on the significant associations found in the main analysis, we further performed an exploratory mediation analysis using the SEM method which failed to detect any mediation effect of neuroimaging measures in the association between plasma NfL and cognitive function. Since a previous study by Mielke et al. ${ }^{7}$ also failed to find cross-sectional association between baseline plasma NfL with cortical thickness and hippocampal volume, the cross-sectional association between plasma NfL and cognitive functions might not be mediated by brain structures. A possible explanation might be that our participants were still in an early phase of neurodegeneration when increased plasma NfL could be found while no obvious brain structural changes could be detected.

We found that plasma NfL was significantly associated with cognitive functions only in the MCI group. Such result is in line with the findings of Mattsson et al. ${ }^{8}$, who studied plasma NfL and cognition in the NC, MCI and $\mathrm{AD}$ populations and reported that plasma NfL was related to longitudinal changes of MMSE only in the MCI adults. A possible explanation for the specific association found only in the MCI group might be that there are larger variances in plasma NfL and cognitive functions in the MCI population than the NC one ${ }^{8,11,12}$, making the plasma NfL association with cognitive functions much easier to be detected.

The large sample size and the longitudinal cognitive data over a 4-year period are the strengths of our study. Notably, the differences of cognitive functions between the NC and the MCI group were bigger in subjects of the Multidomain Alzheimer's Preventive Trial (MAPT) cohort compared to the current study (i.e., cognitive functions of the NC group in the current study were lower than the larger MAPT cohort [Supplementary File Table 1], 


\begin{tabular}{|c|c|c|c|c|c|c|c|c|c|c|}
\hline & \multirow[b]{2}{*}{ Sample size } & \multicolumn{3}{|l|}{ NfL+ group ${ }^{*}$} & \multicolumn{3}{|l|}{ Time } & \multicolumn{3}{|c|}{$\mathrm{NfL}+$ group $\times$ time } \\
\hline & & Coefficient & $p$ & $95 \% \mathrm{CI}$ & Coefficient & $p$ & $95 \% \mathrm{CI}$ & Coefficient & $p$ & $95 \% \mathrm{CI}$ \\
\hline \multicolumn{11}{|l|}{ NC group } \\
\hline MMSE & 219 & -0.35 & 0.21 & $(-0.90,0.19)$ & -0.04 & 0.60 & $(-0.19,0.11)$ & 0.07 & 0.41 & $(-0.10,0.24)$ \\
\hline MMSE orientation & 219 & -0.01 & 0.86 & $(-0.16,0.13)$ & -0.03 & 0.16 & $(-0.07,0.01)$ & -0.01 & 0.61 & $(-0.06,0.04)$ \\
\hline FCSRT & 219 & -0.91 & 0.48 & $(-3.43,1.61)$ & -0.92 & 0.02 & $(-1.72,-0.13)$ & 0.005 & 0.99 & $(-0.89,0.90)$ \\
\hline DSST-WAISR & 219 & -1.18 & 0.49 & $(-4.56,2.20)$ & -0.79 & 0.01 & $(-1.40,-0.17)$ & -0.12 & 0.74 & $(-0.81,0.58)$ \\
\hline Category naming & 219 & -0.07 & 0.96 & $(-2.37,2.24)$ & -0.52 & 0.08 & $(-1.11,0.06)$ & 0.01 & 0.97 & $(-0.65,0.67)$ \\
\hline CCS & 219 & -0.05 & 0.63 & $(-0.23,0.14)$ & -0.10 & $<0.001$ & $(-0.15,-0.05)$ & -0.03 & 0.33 & $(-0.08,0.03)$ \\
\hline \multicolumn{11}{|l|}{ MCI group } \\
\hline MMSE & 277 & -0.42 & 0.15 & $(-0.99,0.15)$ & -0.14 & 0.10 & $(-0.31,0.03)$ & -0.05 & 0.57 & $(-0.24,0.13)$ \\
\hline MMSE orientation & 277 & -0.01 & 0.94 & $(-0.20,0.19)$ & -0.09 & 0.01 & $(-0.15,-0.02)$ & -0.07 & 0.07 & $(-0.14,0.01)$ \\
\hline FCSRT & 277 & -2.23 & 0.22 & $(-5.81,1.34)$ & -1.76 & $<0.001$ & $(-2.51,-1.02)$ & -0.37 & 0.38 & $(-1.20,0.46)$ \\
\hline DSST-WAISR & 277 & -3.62 & 0.004 & $(-6.10,-1.14)$ & -0.94 & $<0.001$ & $(-1.44,-0.43)$ & -0.20 & 0.49 & $(-0.77,0.37)$ \\
\hline Category naming & 277 & -1.70 & 0.13 & $(-3.88,0.49)$ & -0.84 & $<0.001$ & $(-1.32,-0.35)$ & -0.17 & 0.54 & $(-0.72,0.37)$ \\
\hline CCS & 277 & -0.21 & 0.04 & $(-0.42,-0.01)$ & -0.16 & $<0.001$ & $(-0.21,-0.11)$ & -0.07 & 0.02 & $\begin{array}{l}(-0.12 \\
-0.01)\end{array}$ \\
\hline
\end{tabular}

Table 3. Mixed-effects linear analysis of plasma NfL with cognitive functions in stratified NfL quartile subgroups. FCSRT free and cued selective reminding test, DSST-WAISR Digit Symbol Substitution Test score from the Wechsler Adult Intelligence Scale-Revised. "Participants in the non-upper quartile (NfL-) group as the reference.

the CCS in the MCI group was higher in the current study than that in the MAPT [Supplementary File Table 2]). Additionally, we attempted to combine brain imaging data to explore the mediation effect of brain structures on the NfL and cognition relationship. However, this study is limited by the lack of longitudinal plasma NfL data, impeding us from examining a time-matched association between the plasma NfL and cognitive functions. Moreover, since the plasma NfL was tested during the MAPT intervention, we do not know if the interventions have caused any adaptive changes in plasma NfL. Although we have adjusted the MAPT groups in our analysis, further cohort studies with baseline NfL data are needed to generalize our findings to a wider population. Moreover, besides NfL, other cognition-related blood biomarkers, such as brain-derived neurotrophic factor, 3-hydroxykynurenine, lipid levels and total-tau ${ }^{14,15}$, might also be tested as potential candidates in similar analysis to distinguish cognitive status between the NC and the MCI groups. Despite that we found associations between $\mathrm{NfL}$ and global cognitive functions in the MCI group, investigations using a more comprehensive assessment of cognitive functions $s^{7,11,16}$ is needed to confirm our findings.

To conclude, the current study analyzed the association between plasma NfL and cognitive function in nondementia older adults over an up to 4 -year period. We found that the plasma NfL was not associated with cognitive functions in the NC adults while higher plasma NfL levels were associated with lower CCS and more CCS decline in the MCI adults. Our results suggested the possibility of using plasma NfL as a marker of predicting cognitive decline in MCI individuals. Future studies on the mechanism between plasma NfL and cognitive functions are still needed.

\section{Methods}

Study population. Participants in this study came from the randomized controlled trial MAPT (ClinicalTrials.gov [NCT00672685]), which examined 1679 dementia-free older adults (aged $\geq 70$ years). These adults were recruited with any of the following criteria: (1) expressing spontaneous memory complaint, (2) having limitation in at least one instrumental activity of daily, (3) demonstrating slow gait speed (i.e., lower than $0.8 \mathrm{~m} / \mathrm{s}$ ). Participants were excluded if any of the following criteria was met: (1) MMSE score $\leq 24$, (2) diagnosed dementia, (3) having difficulties in basic activities of daily living, (4) taking polyunsaturated fatty acid supplementation. The MAPT tested multidomain interventions (physical activity, nutritional counselling and cognitive training) and omega-3 supplementation, combined or alone, against placebo among older adults and examined changes in cognitive functions over a 3 -year period ${ }^{17}$. The participants were further observationally followed for two additional years, without receiving any intervention. The MAPT was approved by the ethics committee in Toulouse (CPP SOOM II). Written consent forms were obtained from all participants. All research was performed in accordance with relevant guidelines/regulations.

In the current study, participants with extreme plasma NfL levels $(n=5$, over four standard deviations [SDs] above the mean value) or with no Clinical Dementia Rating (CDR, $n=3$ ) score were excluded from an initial group $(n=512)$ in the MAPT that had received plasma NfL tests. Among the 504 participants included in the current study, those with a CDR score of 0.5 were defined as having MCI while others with NC had a CDR score of 0 .

Measurement of plasma NfL. Blood samples were stored in EDTA coated tubes. Plasma neurofilament light chain levels were determined by an electrochemiluminescence-based assay using the R-PLEX human neurofilament L antibody set (F217X-3) with MSD Gold 96-well Small Spot SA SECTOR plates (L45A-1). Samples were diluted twofold in Diluent 12 (R50JA-3) and assayed in duplicate, and read with a Meso Scale Discovery 
instrument. The mean intra-assay coefficient of variation was $7.8 \%$, and the inter-assay coefficient of variation between plates was $15.4 \%$. Over $90 \%$ of the participants $(n=465)$ had plasma NfL tested from blood samples taken 1 year after the enrolment in the study. For the rest of the participants, blood samples from the second year were used. Blood sampling was performed on the same day of cognitive tests.

Measurement of neuroimaging variables. The magnetic resonance imaging (MRI) scan was performed within 12 months after MAPT enrollment ${ }^{17}$. The 3D T1-weighted sequence, derived by the SPM5 toolbox (fil. ion.ucl.ac.uk/spm), was used to measure MRI images. In this study, white matter volume $\left(\mathrm{cm}^{3}\right)$, white matter hyperintensities, hippocampal volume $\left(\mathrm{cm}^{3}\right)$ and amygdala volume $\left(\mathrm{cm}^{3}\right)$ were used for exploratory analysis.

Outcome measures-cognitive functions. Participants completed a comprehensive assessment of four domains: memory (free and total recall of the Free and Cued Selective Reminding Test [FCSRT]), language (the Category Naming Test), executive function (the DSST-WAISR) and orientation (ten MMSE orientation items) ${ }^{18}$.

Global cognitive functions were evaluated as MMSE (ranging from 0 to 30, higher is better) and CCS (mean $z$-scores of the four domains). In the calculation of CCS, the $\mathrm{z}$-score of each domain was calculated using the initial mean and SD values of corresponding test. Original values of each cognitive test and global cognitive functions evaluated at the time-point and after blood tests were included for further analysis.

Covariates. Data of age, sex, body mass index (BMI), education level and MAPT group (i.e., multidomain training + omega-3 supplementation group, multidomain training group, omega-3 supplementation group and control group) were collected and were controlled in the analyses of this study.

Statistics. Descriptive data are presented as mean \pm SD or median [interquartile range] or frequency (percentage), and comparisons between the NC and the MCI groups were performed using Student's t-test, Wilcoxon rank-sum test or chi-squared test as appropriate. Mixed-effects linear models were performed to analyze the cross-sectional and longitudinal associations between plasma NfL and cognitive functions in the whole population and by each cognitive group (i.e., NC and MCI groups). Random effect of participants and random slope of time were assumed.

Within each cognitive group, we further stratified participants into an NfL upper quartile (NfL+) group and a non-upper quartile (NfL-) group with upper quartile NfL values as the cut-off values $(89.7 \mathrm{pg} / \mathrm{ml}$ in the NC group and $93.86 \mathrm{pg} / \mathrm{ml}$ in the MCI group). Similar mixed-effects linear models were performed with stratified NfL groups as an independent variable. Analyses were performed using SAS 9.4 with a two-sided significance level of 0.05 . Result visualization was performed by Python $3.7^{19}$. Due to a large amount of missing values (i.e., $10 \%$ of the present sample), APOE4 was not included in the main model. Sensitive analyses were performed with the APOE genotype as an extra covariate.

Exploratory mediation analysis was performed based on 176 participants (85 MCI participants) with available data using SEM to explore whether the cross-sectional associations between plasma NfL (measured in the first year after the enrollment) and cognitive functions (measured in the first year) were mediated by white matter condition and cognition-related brain structures (measured at a baseline level). Baseline brain imaging neurodegeneration markers (i.e., white matter hyperintensities, white matter volume, hippocampus volume and amygdala volume) were used to create a latent variable of brain degeneration. The analysis was controlled for age, first year BMI and MAPT intervention groups, and was performed in $\mathrm{R}$ (version 4.0.3) 20 $^{20}$. Details of the analysis are included in the supplementary file.

\section{Data availability}

Data in this study are available upon request.

Received: 10 February 2021; Accepted: 12 May 2021

Published online: 28 June 2021

\section{References}

1. Hoffman, P. N. et al. Neurofilament gene expression: a major determinant of axonal caliber. Proc. Natl. Acad. Sci. 84, 3472-3476 (1987).

2. Norgren, N., Rosengren, L. \& Stigbrand, T. Elevated neurofilament levels in neurological diseases. Brain Res. 987, 25-31 (2003).

3. Olsson, B. et al. Association of cerebrospinal fluid neurofilament light protein levels with cognition in patients with dementia, motor neuron disease, and movement disorders. JAMA Neurol. 76, 318 (2019).

4. Skillback, T. et al. CSF neurofilament light differs in neurodegenerative diseases and predicts severity and survival. Neurology $\mathbf{8 3}$, 1945-1953 (2014).

5. Karantali, E., Kazis, D., Chatzikonstantinou, S., Petridis, F. \& Mavroudis, I. The role of neurofilament light chain in frontotemporal dementia: a meta-analysis. Aging Clin. Exp. Res. https://doi.org/10.1007/s40520-020-01554-8 (2020).

6. Khalil, M. et al. Neurofilaments as biomarkers in neurological disorders. Nat. Rev. Neurol. 14, 577-589 (2018).

7. Mielke, M. M. et al. Plasma and CSF neurofilament light: relation to longitudinal neuroimaging and cognitive measures. Neurology 93, e252-e260 (2019).

8. Mattsson, N., Andreasson, U., Zetterberg, H., Blennow, K. \& for the Alzheimer's Disease Neuroimaging Initiative. Association of plasma neurofilament light with neurodegeneration in patients with Alzheimer disease. JAMA Neurol. 74, 557 (2017).

9. Zetterberg, H. \& Blennow, K. From cerebrospinal fluid to blood: the third wave of fluid biomarkers for Alzheimer's disease. JAD 64, S271-S279 (2018).

10. Lu, C.-H. et al. Neurofilament light chain: a prognostic biomarker in amyotrophic lateral sclerosis. Neurology 84, 2247-2257 (2015).

11. Osborn, K. E. et al. Cerebrospinal fluid and plasma neurofilament light relate to abnormal cognition. Alzheimers Dement. (Amst.) 11, 700-709 (2019).

12. Lin, Y.-S., Lee, W.-J., Wang, S.-J. \& Fuh, J.-L. Levels of plasma neurofilament light chain and cognitive function in patients with Alzheimer or Parkinson disease. Sci. Rep. 8, 17368 (2018). 
13. Cullen, N. C. et al. Individualized prognosis of cognitive decline and dementia in mild cognitive impairment based on plasma biomarker combinations. Nat. Aging https://doi.org/10.1038/s43587-020-00003-5 (2020).

14. Küster, O. C. et al. Novel blood-based biomarkers of cognition, stress, and physical or cognitive training in older adults at risk of dementia: preliminary evidence for a role of BDNF, irisin, and the kynurenine pathway. JAD 59, 1097-1111 (2017).

15. Iqbal, G., Braidy, N. \& Ahmed, T. Blood-based biomarkers for predictive diagnosis of cognitive impairment in a Pakistani Population. Front. Aging Neurosci. 12, 223 (2020).

16. Chatterjee, P. et al. Association of plasma neurofilament light chain with neocortical amyloid- $\beta$ load and cognitive performance in cognitively normal elderly participants. JAD 63, 479-487 (2018).

17. Vellas, B. et al. Mapt study: a multidomain approach for preventing Alzheimer's disease: design and baseline data. J. Prev. Alzheimer's Dis. 1, 13-22 (2014).

18. Andrieu, S. et al. Effect of long-term omega 3 polyunsaturated fatty acid supplementation with or without multidomain intervention on cognitive function in elderly adults with memory complaints (MAPT): a randomised, placebo-controlled trial. Lancet Neurol. 16, 377-389 (2017).

19. Van Rossum, G. \& Drake, F. L. Python 3 Reference Manual (CreateSpace, 2009).

20. R Core Team. R: A Language and Environment for Statistical Computing (R Foundation for Statistical Computing, 2020).

\section{Acknowledgements}

The MAPT study was supported by grants from the Gérontopôle of Toulouse, the French Ministry of Health (PHRC 2008, 2009), Pierre Fabre Research Institute (manufacturer of the omega-3 supplement), ExonHit Therapeutics SA, and Avid Radiopharmaceuticals Inc. The promotion of this study was supported by the University Hospital Center of Toulouse. The data sharing activity was supported by the Association Monegasque pour la Recherche sur la maladie d'Alzheimer (AMPA) and the INSERM-University of Toulouse III UMR 1295 Unit.

\section{Author contributions}

L.H. analyzed the data and wrote the manuscript, J.M., G.A. and A.N. performed the NfL analysis, B.V. conceived and designed the experiments, and P.S. guided the analysis and writing. All authors reviewed the manuscript.

\section{Funding}

The present work was performed in the context of the Inspire Program, a research platform supported by grants from the Region Occitanie/Pyrénées-Méditerranée (Reference number: 1901175) and the European Regional Development Fund (ERDF) (Project number: MP0022856), and received additional funds from Alzheimer Prevention in Occitania and Catalonia (APOC Chair of Excellence-Inspire Program).

\section{Competing interests}

The authors declare no competing interests.

\section{Additional information}

Supplementary Information The online version contains supplementary material available at https://doi.org/ 10.1038/s41598-021-91038-0.

Correspondence and requests for materials should be addressed to L.H.

Reprints and permissions information is available at www.nature.com/reprints.

Publisher's note Springer Nature remains neutral with regard to jurisdictional claims in published maps and institutional affiliations.

Open Access This article is licensed under a Creative Commons Attribution 4.0 International format, as long as you give appropriate credit to the original author(s) and the source, provide a link to the Creative Commons licence, and indicate if changes were made. The images or other third party material in this article are included in the article's Creative Commons licence, unless indicated otherwise in a credit line to the material. If material is not included in the article's Creative Commons licence and your intended use is not permitted by statutory regulation or exceeds the permitted use, you will need to obtain permission directly from the copyright holder. To view a copy of this licence, visit http://creativecommons.org/licenses/by/4.0/.

(C) The Author(s) 2021

\section{the MAPT/DSA Group \\ MAPT Study Group \\ Principal investigator \\ Bruno Vellas ${ }^{1,4,5}$ \\ ${ }^{5} \mathrm{CHU}$ Toulouse, Toulouse, France.}


Coordination

Sophie Guyonnet ${ }^{5}$

Project leader

Isabelle Carrié 5

CRA

Lauréane Brigitte ${ }^{5}$

Investigators

Catherine Faisant ${ }^{5}$, Françoise Lala ${ }^{5}$, Julien Delrieu ${ }^{5}$ \& Hélène Villars ${ }^{5}$

Psychologists

Emeline Combrouze ${ }^{5}$, Carole Badufle ${ }^{5}$ \& Audrey Zueras ${ }^{5}$

Methodology, statistical analysis and data management

Sandrine Andrieu ${ }^{5}$, Christelle Cantet ${ }^{5}$ \& Christophe Morin ${ }^{5}$

Multidomain group

Gabor Abellan Van Kan ${ }^{5}$, Charlotte Dupuy ${ }^{5}$, Yves Rolland ${ }^{5}$, Céline Caillaud ${ }^{5}$, Pierre-Jean Ousset ${ }^{5}$, Françoise Lala ${ }^{5}$, Sherry Willis ${ }^{6}$, Sylvie Belleville ${ }^{7}$, Brigitte Gilbert ${ }^{7}$ \& Francine Fontaine $^{7}$

${ }^{6}$ University of Seattle, Seattle, USA. ${ }^{7}$ University of Montreal, Montreal, Canada.

Co-Investigators in associated centres

Jean-François Dartigues ${ }^{8}$, Isabelle Marcet ${ }^{8}{ }_{\text {, Fleur Delva }}^{8}$, Alexandra Foubert ${ }^{8}$, Sandrine Cerda ${ }^{8}$, Marie-Noëlle-Cuffi ${ }^{9}$, Corinne Costes ${ }^{9}$, Olivier Rouaud ${ }^{10}$, Patrick Manckoundia ${ }^{10}{ }^{0}$, Valérie Quipourt ${ }^{10}$, Sophie Marilier ${ }^{10}$, Evelyne Franon ${ }^{10}$, Lawrence Bories ${ }^{11}$, Marie-Laure Pader ${ }^{11}$, Marie-France Basset ${ }^{11}$, Bruno Lapoujade ${ }^{11}$, Valérie Faure ${ }^{11}$, Michael LiYung Tong ${ }^{11}$, Christine Malick-Loiseau ${ }^{11}$, Evelyne Cazaban-Campistron ${ }^{11}$, Françoise Desclaux ${ }^{12}$, Colette Blatge ${ }^{12}$, Thierry Dantoine ${ }^{13}$, Cécile Laubarie-Mouret ${ }^{13}$, Isabelle Saulnier ${ }^{13}$, Jean-Pierre Clément ${ }^{13}$, Marie-Agnès Picat $^{13}$, Laurence Bernard-Bourzeix ${ }^{13}$, Stéphanie Willebois ${ }^{13}$, lléana Désormais ${ }^{13}$, Noëlle Cardinaud ${ }^{13}$, Marc Bonnefoy ${ }^{14}$, Pierre Livet ${ }^{14}$, Pascale Rebaudet ${ }^{14}$, Claire Gédéon ${ }^{14}$, Catherine Burdet ${ }^{14}$, Flavien Terracol ${ }^{14}$, Alain Pesce ${ }^{15}$, Stéphanie Roth ${ }^{15}$, Sylvie Chaillou ${ }^{15}$, Sandrine Louchart ${ }^{15}$, Kristel Sudres ${ }^{16}$, Nicolas Lebrun ${ }^{16}$, Nadège Barro-Belaygues ${ }^{16}$, Jacques Touchon ${ }^{17}$, Karim Bennys ${ }^{17}$, Audrey Gabelle ${ }^{17}$, Aurélia Romano ${ }^{17}$, Lynda Touati ${ }^{17}$, Cécilia Marelli ${ }^{17}$, Cécile Pays ${ }^{18}$, Philippe Robert ${ }^{18}$, Franck Le Duff ${ }^{18}$, Claire Gervais ${ }^{18}$, Sébastien Gonfrier ${ }^{18}$, Yannick Gasnier ${ }^{19}$, Serge Bordes ${ }^{19}$, Danièle Begorre ${ }^{19}{ }$, Christian Carpuat ${ }^{19}$, Khaled Khales ${ }^{19}$, Jean-François Lefebvre ${ }^{19}$, Samira Misbah El Idrissi ${ }^{19}$, Pierre Skoliil ${ }^{19}$ \& Jean-Pierre Salles ${ }^{20}$

${ }^{8} \mathrm{CHU}$ de Bordeaux, Bordeaux, France. ${ }^{9} \mathrm{Hospital}$ of Castres, Castres, France. ${ }^{10} \mathrm{CHU}$ de Dijon, Dijon, France. ${ }^{11}$ Hospital of Foix, Foix, France. ${ }^{12}$ Hospital of Lavaur, Lavaur, France. ${ }^{13}$ University Hospital of Limoges, Limoges, France. ${ }^{14}$ Centre Hospitalier Lyon-Sud, Lyon, France. ${ }^{15} \mathrm{Hospital}$ of Princess Grace, La Colle, Monaco. ${ }^{16} \mathrm{Hospital}$ of Montauban, Montauban, France. ${ }^{17}$ University Hospital of Montpellier, Montpellier, France. ${ }^{18}$ University Côte d'Azur, Nice, France. ${ }^{19} \mathrm{Hospital}$ of Tarbes, Tarbes, France. ${ }^{20} \mathrm{CATI}$ Multicenter Neuroimaging Platform, Gif-sur-Yvette, France.

\section{MRI group}

Carole Dufouil $^{8}$, Stéphane Lehéricy ${ }^{20}$, Marie Chupin ${ }^{20}$, Jean-François Mangin ${ }^{20}$, Ali Bouhayia ${ }^{20}$, Michèle Allard $^{8}$, Frédéric Ricolfi ${ }^{10}$, Dominique Dubois ${ }^{11}$, Marie Paule Bonceour Martel ${ }^{13}$, François Cotton ${ }^{14}$, Alain Bonafé ${ }^{17}$, Stéphane Chanalet ${ }^{18}$, Françoise Hugon ${ }^{19}$, Fabrice Bonneville ${ }^{5}$, Christophe Cognard ${ }^{5}$ \& François Chollet ${ }^{5}$ 
PET scans group

Pierre Payoux ${ }^{5}$, Thierry Voisin ${ }^{5}$, Julien Delrieu ${ }^{5}$, Sophie Peiffer ${ }^{5}$, Anne Hitzel ${ }^{5}$, Michèle Allard ${ }^{8}$, Michel Zanca $^{17}$, Jacques Monteil ${ }^{13}$ \& Jacques Darcourt ${ }^{18}$

Medico-economics group

Laurent Molinier ${ }^{5}$, Hélène Derumeaux ${ }^{5} \&$ Nadège Costa $^{5}$

Biological sample collection

Bertrand Perret $^{5}$, Claire Vinel ${ }^{5}$ \& Sylvie Caspar-Bauguil ${ }^{5}$

Safety management

Pascale Olivier-Abbal ${ }^{5}$

DSA Group

Sandrine Andrieu ${ }^{5}$, Christelle Cantet $^{5}$ \& Nicola Coley ${ }^{5}$ 